\title{
ATTACK ON LIU/FARRELL/BOYD ARITHMETIC CODING ENCRYPTION SCHEME
}

\author{
Takeyuki Uehara, Reihaneh Safavi-Naini \\ School of Information Technology and Computer Science, \\ University of Wollongong, Northfields Ave \\ Wollongong, NSW 2522 - AUSTRALIA \\ tu01Quow.edu.au \\ reiQuow.edu.au
}

\begin{abstract}
Arithmetic coding is an optimal data compression algorithm. Witten and Cleary proposed incorporating security into arithmetic coding. This has been an attractive proposal that could result in less overall processing overhead compared to the traditional method of compression followed by encryption. However a number of attacks on arithmetic coding encryption systems have been published which demonstrate the difficulty of successful combination of the two processes. Recently Liu/Farrell/Boyd proposed a new scheme which claimed to be resistant against all the known attacks. In this paper we study the security of this scheme and show an attack that can recover the plaintext message and whose cost is $2^{20}$.
\end{abstract}

Keyword: data compression, encryption, arithmetic coding

\section{INTRODUCTION}

Arithmetic coding is an optimal data compression algorithm that compresses input data on-the-fly and so introduces minimum delay during the compression phase. Because of the optimality and small delay arithmetic coding has been widely used in numerous applications such as JPEG compression system [ITU93] which is a widely adopted image compression algorithm. In arithmetic coding an encoded message is represented by an interval within the interval $[0,1)$ [BWC90]. Encoding process starts with an initial interval which is narrowed down with the encoding of the new incoming symbols such that the amount of narrowing is determined by the probability of the coded symbol. In adaptive 
arithmetic coding schemes the model is updated by the incoming symbols. Arithmetic coding encryption schemes were proposed by Witten and Cleary [WC88] in an attempt to combine encryption with compression systems. This has been an attractive proposition as it could potentially result in more efficient systems.

An arithmetic coder consists of two main parts: a model that captures the statistical property of the input data, and a coder which produces the output. Witten et al [WC88] noted that if the model is kept secret and only known to the intended receiver, then other receivers without the knowledge of the model cannot recover the compressed message. This is called a model-based scheme. In adaptive systems the model is continuously updated with the incoming symbols and so the coded output depends on the whole input which could imply that it is very difficult for an eavesdropper to track the whole input and recover the message. However adaptive model-based schemes were shown to be vulnerable to chosen plaintext attacks [Irv95] or to an attacker who has access to an arithmetic coder device and can repeatedly reset the system [LBD97]. A second type of arithmetic coding encryption system, called coder-based system, was proposed by Irvine et al. [ICRM95]. In these systems, the coder parameters were used as the key information. Attack on coder-based schemes were published in [ICRM95] and [USN99]. In [LFB97], Liu/Farrell/Boyd proposed a combination of model and coder based scheme, that we call LFB system for short, and estimated the cost of the best known attack to be $2^{79}$. In this paper we show an attack on the LFB system which requires approximately $2^{20}$ steps and hence shows that the system is completely insecure under the new proposed attack.

The paper is organized as follows. In Section 2, we review an implementation of arithmetic coding systems and then describe arithmetic encoding encryption schemes and Bergen/Hogan attack on model-based schemes. In Section 3, we describe the details of our attack. Finally in Section 4 we summarize our results.

\section{PRELIMINARIES}

In this section we review an implementation of an arithmetic coding scheme and briefly describe arithmetic coding encryption schemes and Bergen/Hogan attack which is the basis of our attack. 


\subsection{IMPLEMENTATION OF ARITHMETIC CODING}

A software implementation of an arithmetic coding was given by Witten/Neal/Cleary (WNC implementation ) [WNC87]. The implementation is order- 0 adaptive arithmetic encoder/decoder in which the model consists of the symbols' frequencies. The model is implemented by an ordered frequency table where the symbol with the highest frequency appears at the top and other symbols are listed in decreasing frequency order. A symbol $\psi$ is associated with a frequency, $F(\psi)$, and a cumulative frequency, $C(\psi)$, which is the sum of the symbol's frequency and the frequencies of symbols below it. There are 256 symbols corresponding to all binary 8 bit strings. Adaptiveness is achieved by incrementing $F(\psi)$ by one when $\psi$ is received in the input. In this case, $C(\psi)$ and $F(\psi)$, together with $C\left(\psi^{\prime}\right)$ for all $\psi^{\prime}$ above $\psi$ are incremented. If this results in a violation of the order, $\psi$ is moved up in the table so that the frequency order is maintained. Let $C_{\max }$ denote maximum value of $C(\psi)$, and $\psi_{\max }$ and $\psi_{\min }$ denote symbols at the top and at the bottom of the table with maximum and minimum frequencies, respectively. If $C\left(\psi_{\max }\right)=C_{\max }$, then halving occurs and all frequencies are divided by 2 ; that is

$$
F(\psi)=\left\lfloor\frac{F(\psi)+1}{2}\right\rfloor
$$

for all $\psi$ s. $F(\psi)$ is maximized when $C(\psi)=C_{\max }$ and frequencies of all other symbols $\psi^{\prime}$ satisfy $F\left(\psi^{\prime}\right)=1$. In this case, $F(\psi)$ is given by

$$
F(\psi)=C_{\max }-N_{\text {symbol }}+1
$$

where $N_{\text {symbol }}$ is the number of symbols. If another $\psi$ is sent, halving occurs and from (1) and (2), the frequency $F(\psi)$ is given by

$$
F(\psi)=\left\lfloor\frac{C_{\max }-N_{\text {symbol }}+1}{2}\right\rfloor+1
$$

The coding procedure is realized using finite precision integer arithmetic. The interval is represented by two 16 bit numbers representing the high and the low values. The values are updated based on model probabilities and the output consists of the matched part of the high and low values. With the $i^{\text {th }}$ incoming symbol, $\psi$, the new high and low values are calculated as

$$
h_{i}^{\prime}=l_{i}+\left\lfloor\frac{\left(h_{i}-l_{i}\right) C(\psi)}{C\left(\psi_{\max }\right)}\right\rfloor-1 \text { and } l_{i}^{\prime}=l_{i}+\left\lfloor\frac{\left(h_{i}-l_{i}\right) C\left(\psi^{\prime}\right)}{C\left(\psi_{\max }\right)}\right\rfloor
$$

where $\psi^{\prime}$ is the next symbol below $\psi$ in the table. If $h_{i}^{\prime}$ and $l_{i}^{\prime}$ have $u$ common bits in the most significant bit positions, the following steps will be executed $u$ times: 
1. The most significant bit of $h_{i}^{\prime}$ which is the same as the most significant bit of $l_{i}^{\prime}$, is sent to the output and $h_{i}^{\prime}$ and $l_{i}^{\prime}$ are left-shifted.

2. If the interval satisfies $0 \times 4000 \leq$ interval $<0 \times \mathrm{x} 000$, mid-range buffering is used. In this case, $0 \times 4000$ is subtracted from both values, the two values are left-shifted and one bit, which is still undetermined, is stored in a mid-range buffer called bits_to_follow.

3. When the interval is in the mid-range, the output bit is 1 when the following output bit is 0 and 0 when the following output bit is 1 . and so the bit is determined when the next output bit is produced.

Define two functions $S_{0}(x, u)$ which shifts $x$ to the left by $u$ bits and fills the least significant $u$ bits by 0 , and $S_{1}(x, u)$ which shifts $x$ to the left by $u$ bits and fills the least significant $u$ bits by 1 . Assuming that $h_{i}^{\prime}$ and $l_{i}^{\prime}$ produce the output $o_{i}$ and the size of $o_{i}$ is $u$ bits, the resulting high and low values are as follows

$$
\begin{aligned}
& h_{i}^{\prime \prime}=S_{1}\left(h_{i}^{\prime}, u\right) \text { and } l_{i}^{\prime \prime}=S_{0}\left(l_{i}^{\prime}, u\right)
\end{aligned}
$$

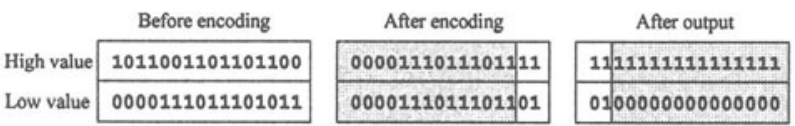

Figure 1 Output of the high and low values

\subsection{ARITHMETIC CODING ENCRYPTION SCHEMES}

In Witten and Cleary's proposals the model is used as the encryption key: that is the details of the model is only known to the transmitter and the receiver. The schemes are called model-based. An alternative approach proposed by Irvine, Cleary and Rinsma-Melchert is coder-based scheme in which the coder's range, consisting of the high and the low values, is shared by transmitter and receiver and is kept secret from others [ICRM95].

\section{Model-based scheme 1}

Use the initial model as the key. Witten and Cleary suggested an array of single-character frequencies in the range of 1-10. In this scheme, the initial range for the coder is public.

\section{Model-based scheme 2}


Use an initial string as the key. The initial model and range are public. Prior to encrypting a message, a secret initial string shared by transmitter and receiver is used to modify the model and the range.

\section{Coder-based scheme}

The key is a bit string which is used to narrow the range. Based on a key bit, either the high value $h$ is decreased or the low value $l$ is increased by the amount $(h-l) \varepsilon$ where $\varepsilon$ is a public parameter and $0<\varepsilon<1$. A variation of this scheme, proposed in [LFB97], has two pairs of secrets, $\left(\varepsilon_{l}, \varepsilon_{h}\right)$, which are selected based on the key bit.

\subsection{BERGEN/HOGAN ATTACK ON ADAPTIVE SYSTEMS}

Bergen and Hogan [BH93] proposed an attack on the model-based adaptive arithmetic coding encryption schemes. The attack is a chosen plaintext attack where the attacker can feed plaintexts of her/his choice to the encoder. The attack does not discover the key (initial model) but succeeds in modifying the model into a form which is known to the attacker, hence allowing the attacker to decrypt the communication afterwards.

The assumptions of the attack are as follows.

- The system is a model-based scheme of first or second type. That is, the key is either initial model or the initial string. The initial state of the encoder does not affect the attack.

- The system uses order- 0 adaptive arithmetic coding. The attack is based on WNC implementation .

- The attacker is able to send symbols to the encoder and obtain the output.

- The attacker has a decoder.

This attack uses two properties of the WNC implementation : halving of symbols' frequencies when $C\left(\psi_{\max }\right)=C_{\max }$, and the ordering that is imposed on the frequency table. The attacker sends a long string of a single symbol, $\psi$, to cause consecutive halving. If the string is chosen long enough and enough number of halvings occurs, $\psi$ eventually moves to the top of the table and frequencies of all other symbols become 1 . If more $\psi$ are sent so that another halving occurs, the frequency of $\psi$ will become a constant, given by

$$
C(\psi)=\left\lfloor\frac{C_{\max }-N_{\text {symbol }}+1}{2}\right\rfloor+1 .
$$


We refer to this state of the model as the synchronized state. We note that once the model is synchronized the number of $\psi$ required to produce another halving, denoted by $n$, becomes a constant

$$
n=C_{\max }-N_{\text {symbol }}-\left\lfloor\frac{C_{\max }-N_{\text {symbol }}+1}{2}\right\rfloor .
$$

At this stage the attacker does not know the order of the symbols in the table. He/She sends other symbols one by one, each time increasing the frequency $F(\psi)$ from 1 to 2 , moving the sent symbol above other symbols with frequency 1 , and hence effectively re-ordering the frequency table. If halving of frequencies occurs during the re-ordering procedure, re-ordering fails and re-ordering must be repeated.

Now the model can be verified by decoding a message. We note that for correct decoding the attacker must know the range of the coder at this stage.

\subsection{LIU/FARRELL/BOYD SCHEME}

Liu/Farrell/Boyd (LFB) proposed a system that combines the modelbased and the coder-based schemes [LFB97]. The secret key of the scheme consists of the following elements.

1. The initial model.

2. The initial range in the coder. The range should be larger than $\frac{\left(2^{16}-1\right)}{4}$.

3. A 16 bit substitution that is used to substitute the first 16 bits of the encoder's output.

4. Two sets of shrinking factors $\left(\varepsilon_{h_{0}}, \varepsilon_{l_{0}}\right)$ and $\left(\varepsilon_{h_{1}}, \varepsilon_{l_{1}}\right)$, each consisting of an upper and a lower shrinking factors. This is used to narrow the range after encoding a symbol. The shrinking factors $\varepsilon_{h_{j}}$ and $\varepsilon_{l_{0}}$ should satisfy $\varepsilon_{h_{0}} \neq \varepsilon_{h_{1}}$ and $\varepsilon_{l_{0}} \neq \varepsilon_{l_{1}}$ and $0.9000 \leq$ $\varepsilon_{h_{j}} \leq 0.9999$ and $0.0000 \leq \varepsilon_{l_{j}} \leq 0.0999$ where $j=\{0,1\}$.

5. A 128 bit random sequence that is cyclically used and whose elements determine which one of the two sets of shrinking factors is to be used.

The procedure to encode a symbol is as follows.

Step 1 Encode a symbol according to (4).

Step 2 Output a bit sequence. The high and low values are calculated as shown in (5).

Step 3 Shrink the range. 


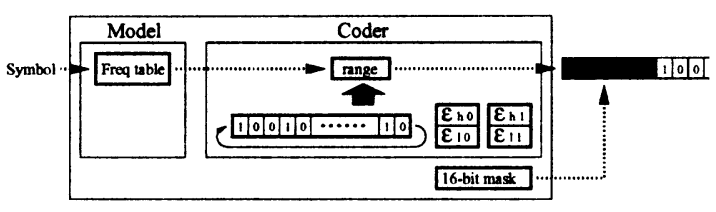

Figure 2 LFB scheme

For a set of shrinking factors, $\left(\varepsilon_{h_{j}}, \varepsilon_{l_{j}}\right), j=0,1$, the new $h_{i+1}$ and $l_{i+1}$ are calculated as follows.

$$
h_{i+1}=l_{i}^{\prime \prime}+\left(h_{i}^{\prime \prime}-l_{i}^{\prime \prime}\right) \varepsilon_{h_{j}} \quad \text { and } \quad l_{i+1}=l_{i}^{\prime \prime}+\left(h_{i}^{\prime \prime}-l_{i}^{\prime \prime}\right) \varepsilon_{l_{j}}
$$

The value of $j$ is determined by the 128 bit random sequence.

The scheme results in approximately $2 \%$ drop in the compression rate and almost doubles the processing time. LFB claimed that the scheme is resistant against $\mathrm{BH}$ attack and estimated the cost of the attack to be $2^{14} \times 2^{30} \times 2^{16} \times 2^{19}=2^{79}$.

\section{ATTACK ON LIU/FARRELL/BOYD SCHEME}

In this section we describe an attack on the LFB scheme. The attack has two steps and its cost is $2^{20}$. In the first step the attacker uses BH attack to take control over the model. This is by sending a chosen message and finding an approximate value for the halving point. In the second step he uses a method of discovering the shrinking factors by encoding symbols of small frequencies.

\subsection{OUTLINE OF THE ATTACK}

The relationship between an input symbol, the model and the coder is i) the model is modified according to an input symbol and ii) the coder encodes a symbol, which is represented by two consecutive cumulative frequencies given by the model. Once the model is known, the coder can be controlled using this knowledge. $\mathrm{BH}$ attack modifies the model into a known form. The weakness of $\mathrm{BH}$ attack is that if halving occurs during the re-ordering procedure, the attack fails. Our proposed attack strengthens $\mathrm{BH}$ attack by enabling the attacker to have a good estimate of the halving point. If the model is known, the secret parameters of the coder, $h_{i}$ and $l_{i}$, can be closely approximated by encoding symbols of small frequencies. From (5), when output size is $u$ bits, the number of unknown bits after output is $16-u$. This is because the least significant $u$ bits are filled with the known values. If $u$ is large, the number of the unknown bits becomes small. The maximum of $u$ is given by 


$$
-\log _{2} \frac{1}{C_{\max }}=-\log _{2} \frac{1}{16383} \approx 14
$$

and hence, the unknown bits of $h_{i}$ and $l_{i}$ are reduced to 2 . Using the knowledge of the model it is possible to selectively encode symbols of small frequencies. Furthermore, it is known that $C\left(\psi_{\max }\right)$ is maximized right before halving.

The assumptions are as follows.

1. The implementation is based on WNC implementation. The output is byte-buffered, ie. 8 bit blocks are output. We do not consider any I/O buffering of stdio, which may be used by the operating system since the scheme does not explicitly take into account the blocking and the buffering as part of the system. The effect of the blocking on the attack is discussed in Section 3.4.

2. The attack is a chosen plaintext attack. The attacker has access to the encoder and can see the output. The attack does not require any reset of the system.

The steps of the attack are

Step 1 i) Over-flood the model by sending a long enough message consisting of a single symbol $\psi$ and observe the variation of the output length per symbol. Obtain a rough estimate of the halving point. Details of how to detect the halving point is described in [USN99].

ii) Re-order the symbols in the frequency table. At this stage, the model becomes known to the attacker. That is, the frequencies of all the symbols except $\psi$ at the top of the table become 2 .

Step 2 i) Send a message consisting of the symbol $\psi$ again to cause a halving and to make $C(\psi)$ close to $C_{\max }$, ie. 16383. After this, the frequencies of all the symbols except $\psi$ at the top of the table become 1 again.

ii) Repeatedly send the symbols $\psi_{\min }$ at the bottom of the frequency table and obtain the output. There are 255 symbols with frequency 1 . Encoding these symbols produces a considerably long output. Details are given in Section 3.3.

iii) Identify the output bit sequence for each of the 255 symbols by analyzing the bit patterns. Details are given in Section 3.4.

iv) Calculate the lower shrinking factors from the bit sequence. Details are given in Section 3.3.

v) Find the 128 bit random sequence using the lower shrinking factors found. 
In the following, we analyze the security provided by the shrinking factors and give a method to find the shrinking factors and hence the random bit sequence.

\subsection{SECURITY OF THE SHRINKING FACTORS}

Assumptions are as follows.

1. The model is known. This means that the method will be applied after synchronization of the model.

2. $C\left(\psi_{\max }\right)$ is close to $C_{\max }$, ie. 16383 .

3. The frequencies of the symbols other than the one at the top of the table are 1 .

4. The start of the output sequence corresponding to input symbols is known.

5. There is no mid-range buffering.

When a symbol with a probability close to $\frac{1}{C_{\max }}$, is encoded, if there is no shrinking, the output length is approximately 14 bits. This follows from (9). The extra-narrowing shrinks the range by at most approximately $80 \%$ and so the output length will be $-\log _{2} 0.8 \frac{1}{C_{\max }} \approx 14.3$ bits. This affects the required number of bits for a symbol. For example, right before halving and without shrinking, the required number of bits for $\psi_{\max }$ is $-\log _{2} 0.9844$. If the range is shrinked by $80 \%$, the required length is $-\log _{2}(0.9844 \times 0.8)=0.3446$ bits. The extra-narrowing results in two possible ranges for each symbol and one of them will be selected depending on the pseudo-random bit. Although for a single character, the extra-narrowing can disturb detection of the halving point but if the average length over blocks of 128 characters is calculated, the variation in the range can be averaged and the halving point can be detected.

From (5), if the output size is 14 bits, the high and low values are $h_{i}^{\prime \prime}=$ $* * 11111111111111$ and $l_{i}^{\prime \prime}=* * 00000000000000$ respectively. Following the output production procedure in Section 2.1, we note that $i) h_{i}^{\prime \prime}$ and $l_{i}^{\prime \prime}$ do not have any common most significant bits and ii) the interval defined by $h_{i}^{\prime \prime}$ and $l_{i}^{\prime \prime}$ does not satisfy $0 \times 4000 \leq$ interval $<0 \times 0000$. There are three pairs of $h_{i}^{\prime \prime}$ and $l_{i}^{\prime \prime}$ which satisfy these conditions; these possible pairs are, (0xFFFF, 0x4000), (0xFFFF, 0x0000) and (0xBFFF, 0x0000).

$h_{i}^{\prime \prime}$ and $l_{i}^{\prime \prime}$ are narrowed down by either of the two sets of shrinking factors according to the 128 bit random sequence. Hence, there are six 
possible ranges in Step 1 of Section 2.4. Each of the six ranges becomes one of the three possible ranges after the output procedure in Step 2 in Section 2.4 .

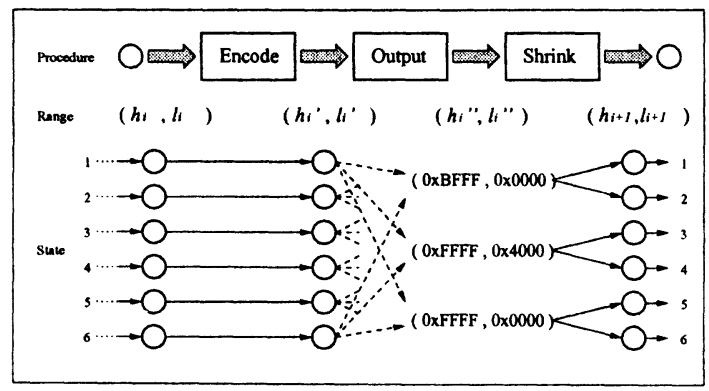

Figure 3 One cycle of encoding procedure

3.2.1 Weakness in the coder . Assume $o_{i}$ is a 16 bit value, where its $14 \mathrm{MSB}$ are the output bits and the rest are 0, and assume there is no mid-range buffering. Then, $o_{i}$ is very close to $l_{i}^{\prime}$ because $\frac{h_{i}-l_{i}}{C\left(\psi_{\max }\right)}$, given in (4), is very small. So the attacker can derive $l_{i}^{\prime}$ from the output $o_{i}$ if a symbol with small probability is encoded and midrange buffering does not exist. The high and low values in Step 2 of Section 2.4 are known by the attacker.

\subsection{A METHOD TO DERIVE THE SHRINKING FACTORS FROM THE OUTPUT}

From (4) in Step 2 of Section 3., the high and low values $h_{i}^{\prime}$ and $l_{i}^{\prime}$, after encoding a symbol $\psi_{\min }$ is calculated as,

$$
h_{i}^{\prime}=l_{i}+\frac{2\left(h_{i}-l_{i}\right)}{C\left(\psi_{\max }\right)} \quad \text { and } \quad l_{i}^{\prime}=l_{i}+\frac{h_{i}-l_{i}}{C\left(\psi_{\max }\right)}
$$

where $C\left(\psi_{\max }\right)$ is close to $C_{\max }$.

From (8) and (10),

$$
\begin{aligned}
h_{i+1}^{\prime} & =l_{i+1}+\frac{2\left(h_{i+1}-l_{i+1}\right)}{C\left(\psi_{\max }\right)+1}=l_{i}^{\prime \prime}+\left(h_{i}^{\prime \prime}-l_{i}^{\prime \prime}\right) \varepsilon_{l j}+\frac{2\left(h_{i}^{\prime \prime}-l_{i}^{\prime \prime}\right)\left(\varepsilon_{h j}-\varepsilon_{l j}\right)}{C\left(\psi_{\max }\right)+1} \\
l_{i+1}^{\prime} & =l_{i+1}+\frac{h_{i+1}-l_{i+1}}{C\left(\psi_{\max }\right)+1}=l_{i}^{\prime \prime}+\left(h_{i}^{\prime \prime}-l_{i}^{\prime \prime}\right) \varepsilon_{l j}+\frac{\left(h_{i}^{\prime \prime}-l_{i}^{\prime \prime}\right)\left(\varepsilon_{h j}-\varepsilon_{l j}\right)_{11}}{C\left(\psi_{\max }\right)+1}
\end{aligned}
$$

When $C\left(\psi_{\max }\right)$ is close to 16383 , the attacker knows that the length of the encoder's output is 14 bits and that the high and low values, $h_{i}^{\prime \prime}$ 
and $l_{i}^{\prime \prime}$, take one of the three values given in Section 3.2. Let the three ranges, (0xBFFF, 0x0000), (0xFFFF, 0x4000) and (0xFFFF, 0x0000) be called Pattern 1, Pattern 2 and Pattern 3, respectively.

Pattern $1 h_{i}=0 \times \mathrm{xBFF}, l_{i}=0 \times 0000$,

$$
\begin{aligned}
h_{i+1}^{\prime} & =\operatorname{OxBFFF} \varepsilon_{l j}+\frac{\operatorname{Ox} 17 \mathrm{FFE}\left(\varepsilon_{h j}-\varepsilon_{l j}\right)}{16383} \\
l_{i+1}^{\prime} & =\operatorname{OxBFFF} \varepsilon_{l j}+\frac{\operatorname{OxBFFF}\left(\varepsilon_{h j}-\varepsilon_{l j}\right)}{16383}
\end{aligned}
$$

From (8),

$$
\begin{aligned}
& 0.8001 \leq \quad \varepsilon_{h j}-\varepsilon_{l j} \quad \leq 0.9999 \\
& 4 \leq \frac{\operatorname{Ox} 17 \mathrm{FFE}\left(\varepsilon_{h j}-\varepsilon_{l j}\right)}{16383} \leq 5 \\
& \frac{\operatorname{OxBFFF}\left(\varepsilon_{h j}-\varepsilon_{l j}\right)}{16383}=2
\end{aligned}
$$

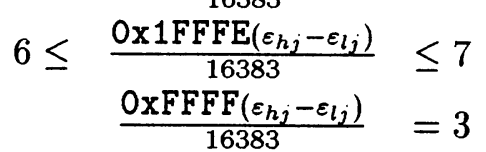

Similarly, $h_{i+1}^{\prime}$ and $l_{i+1}^{\prime}$ for Pattern 2 and Pattern 3 can be calculated. From (12), (13) and the calculation of Pattern 2 and Pattern $\mathbf{3}$, it can be seen that the effect of $\varepsilon_{h j}-\varepsilon_{l j}$ is small.

Define $\alpha$ as $\frac{\beta\left(\varepsilon_{h_{j}}-\varepsilon_{l_{j}}\right)}{16383}$ where $\beta=\{0 \times 1$ FFFE, Ox17FFE, OxFFFF, OxBFFF $\}$ and assume that there is no mid-range buffering, and $o_{i+1}$ is a 16 bit value, where its most significant 14 bits is the output and the rest is 0 . Because the output $o_{i+1}$ is very close to $l_{i+1}^{\prime}, \varepsilon_{l j}$ can be shown as follows.

Pattern $1 h_{i}=0 \times \mathrm{xBFF}, l_{i}=0 \times 0000$,

$$
o_{i+1} \approx 0 \times \mathrm{xBFFF} \varepsilon_{l j}+\alpha \text { and } \varepsilon_{l j} \approx \frac{o_{i+1}-\alpha}{0 \mathrm{xBFFF}}
$$

From (13), $\frac{\alpha}{0 \times F F F F} \leq 0.0001$ and $\frac{\alpha}{0 \times B F F F} \leq 0.0001$. So the lower shrinking factor $\varepsilon_{l j}$ can be accurately calculated from the output.

There are three possible patterns of the high and low values and there may be 0.0001 error in the calculation of the lower shrinking factor. So the cost of obtaining the lower shrinking factor is $3 \times 2$. Notice that $o_{i+1}$ in Pattern 2 is equal to $o_{i+1}+0 \times 4000$ in Pattern 1.

Now the lower shrinking factor $\varepsilon_{l j}$ is given and from (12), the upper shrinking factor can be calculated as follows.

Pattern $1 h_{i}=0 \times \mathrm{BFFF}, l_{i}=0 \times 0000$,

$$
\begin{aligned}
\varepsilon_{h j} & =\varepsilon_{l j}+\frac{16383}{0 \times \mathrm{XBFF}}\left(l_{i+1}^{\prime}-0 \mathrm{XBFFF} \varepsilon_{l j}\right) \\
& \approx \varepsilon_{l j}+\frac{16383}{0 \mathrm{XBFFF}}\left(o_{i+1}-0 \mathrm{XBFFF} \varepsilon_{l j}\right)
\end{aligned}
$$


However, the terms $\frac{16383}{0 \times \mathrm{BFFF}}$ and $\frac{16383}{0 \times \mathrm{FFFF}}$ are very small and $o_{i+1}$ is an approximation of $l_{i+1}^{\prime}$. This means that the accurate upper shrinking factor cannot be found by this calculation and to find the upper shrinking factor, a brute-force method must be used. The cost of the brute force attack is approximately $2^{10}$ because the unknown factor is between 0.9000 and 0.9999 .

\subsection{ANALYSIS OF THE OUTPUT BIT SEQUENCE}

To derive the shrinking factors from the output, it is necessary to identify the output bit sequence for a symbol in the whole output. This section analyzes the output bit sequence in terms of the relationship between an encoded symbol and its resulting output bit sequence, and shows a method of identifying the bit sequence produced by encoding a symbol.

There are two factors which hide the direct relationship between an encoded symbol and its output bit sequence. One is the byte buffering, that is, 8 bit blocking of output, and the second is mid-range buffering. Byte buffering merges consecutive encoding results and is required for the file $\mathrm{I} / \mathrm{O}$ in the software implementation. It delays the output but does not change the sequence of the output bits. If the mid-range buffer, as a result of encoding a symbol $\psi_{i}$, contains $n$ bits, then $n$ bits are inserted between the first and the second bit of the output of the symbol $\psi_{i+1}$. These $n$ bits are opposite to the first bit, ie. if the first bit is 0 , the $n$ bits are all 1 and if it is 1 , the $n$ bits are all 0 . Hence it is necessary to identify the inserted bits to correctly derive the low value from the output.

3.4.1 Method to identify the output bit sequence . After synchronization of the model, assume that the total frequency is close to $C_{\max }$, ie. 16383 , and symbols of frequency $=1$ are encoded. The following properties of the output bit sequence are useful in finding the beginning of a symbol in the output.

1. If the first two bits are the same, there is no mid-range buffering. This is because the inserted $n$ bits are always opposite of the first bit.

2. The first bit of the output is always 0 .

When the symbol at the bottom of the frequency table is encoded, the resulting output is close to the low value and hence the first bit is 0 . 
The method to find the beginning of the bit sequence for a symbol is as follows.

1. Choose a bit 0 in the output and assume it is the beginning of the sequence.

If the length of the output for a symbol is $14 \mathrm{bits}$, there is a start bit in any 14 consecutive bits of the output. If $\gamma$ bits are 0 , there are $\gamma$ possible start bits.

2. If the bit after the chosen bit is also 0 , there has been no mid-range buffering.

Notice that since a 14 bit sequence includes a start bit, there must be at least one 0 .

3. If $\sigma$ bits after the chosen bit are 1 , the first $i$ bits, $0 \leq i \leq \sigma$, of $\sigma$ bits may be the result of mid-range buffering. Remove $i$ bits from the output and construct the sequence without mid-range buffering.

4. Assume that the sequence of 14 bits without mid-range buffering obtained above is the output for a symbol and calculate the lower shrinking factor. Using the method given in Section 3.3, calculate the value of lower shrinking factor for each possible range.

Since there are 3 possible ranges, 3 calculations per symbol is required to obtain the lower shrinking factor. The factor may include error but it is no larger than 0.0001 . Hence the cost to find the factor is $3 \times 2$.

5. Verify the result using an encoder. Encode a symbol of frequency $=$ 1 while using a total frequency close to 16363 and the lower shrinking factor obtained in the above step. Compare the result with the original bit sequence. If the shrinking factor is correct, it should produce exactly the same sequence as the original.

If it produces a different sequence, it is either because the assumption of the start bit is wrong or the shrinking factor is wrong. Then change $i$ in step 3 and repeat the above procedure.

From our experiments, for the sequence produced by encoding symbols at the bottom of the frequency table, the number of mid-range buffered bits is usually small. Hence the output produced by encoding one of these symbols, including mid-range buffering, will not be much larger than 14 bits. If there are $\gamma$ possible start bits, ie. Os, and each of them produces $\beta_{j}, 1 \leq j \leq \gamma$, possible bit sequences because of the 
removing of the possible mid-range buffered bits, there are $\sum_{j=1}^{\gamma} \beta_{j}$ possible binary sequences for a symbol. Each sequence is verified by the above procedure. When the sequence for the first symbol is determined, it automatically determines the bit sequence for the next symbol in the output and results in a chain of possible bit sequences. If the next sequence does not satisfy the conditions, ie. $i$ ) it does not start with 0 , or ii) the mid-range buffered bits that are inserted into the output of the next symbol result in different number of zeros and ones, or finally iii) applying the shrinking factor does not result in the expected sequence, then the sequence is wrong. In this case the branch is pruned and does not grow further.

Once one of the lower shrinking factors is found, the 128 bit random sequence can be found by encoding symbols of small frequencies in a way similar to that used in the verification of the result. By using the lower shrinking factor to encode the symbols, all parameters, including the high and low values and the size of the mid-range buffering can be found. If the experimental results differ from the original sequence, the analysis will be repeated with the following starting point in the input sequence. Once the two lower shrinking factors are known, the 128 bit random sequence can be found by applying one of the two shrinking factors.

\subsection{COST OF THE ATTACK}

The cost of finding the start of output bit sequence for a symbol is at most $14 \approx 2^{3.8}$ and the cost of calculating the lower shrinking factor is $3 \times 2 \approx 2^{2.6}$. To verify the lower shrinking factor, it is necessary to encode a symbol using the upper shrinking factor, which is unknown. However, the influence of the upper shrinking factor is very small and so it is sufficient to only try the maximum, ie. 9999, the minimum, ie. 9000 , and a few values in between. The total cost is $2^{6.4}+\theta$ where $\theta$ is the cost for the verification.

The unknown part of the key is only the two upper shrinking factors, each of which could take a value from 0.9000 to 0.9999 . Hence, the attack reduces the unknown key to $2^{10} \times 2^{10}=2^{20}$ trials.

\section{CONCLUSION}

We have shown that LFB scheme cannot provide the claimed level of security. An important assumption in this scheme is that if the coder is randomized and hence remain unknown, the attacker cannot verify the result of encoding. We showed that if the halving point is known, it is possible to guarantee successful re-ordering of the symbols in the 
frequency table, and that if the model is known the attacker can control the coder and is able to modify the coder into a known state.

This shows that $\mathrm{BH}$ attack can effectively synchronize the encoder and the decoder in the middle of the transmission disregarding the effect of the initial state of the encoder (the initial model and the initial coder range).

\section{References}

[BH93] Helen A. Bergen and James M. Hogan. A chosen plaintext attack on an adaptive arithmetic coding compression algorithm. Computers and Security, 12(2):157-167, 1993.

[BWC90] T. C. Bell, I. H. Witten, and J. G. Cleary. Text Compression. Prentice-Hall, 1990.

[ICRM95] Sean A. Irvine, John G. Cleary, and Ingrid Rinsma-Melchert. The subset sum problem and arithmetic coding . http://www.cs.waikato.ac.nz/ sirvine/, Sep 1995.

[Irv95] Sean A. Irvine. Compression and Cryptology. PhD thesis, School of Computing and Mathematical Sciences, University of Waikato, 1995.

[ITU93] ITU. ITU-T T.81 /ISO/IEC 109181 - JPEG standard text (postscript). http://icib.igd.fhg.de/icib/telecom/ccitt/rec_t.81/scan.html, 1993.

[LBD97] Jen Lim, Colin Boyd, and Ed Dawson. Cryptanalysis of adaptive arithmetic coding encryption schemes. ACISP, pages 216-227, 1997.

[LFB97] Xian Liu, Patrick G. Farrell, and Colin Boyd. Resisting the bergen-hogan attack on adaptive arithmetic coding. LNCS1355, Cryptography and Coding, Springer, pages 199-208, Dec 1997.

[USN99] Takeyuki Uehara and Reihaneh Safavi-Naini. Attacking and mending arithmetic coding encryption schemes. Australian Computer Science Communications, Springer, 21(1):408419, Jan 1999.

[WC88] Ian H. Witten and John G. Cleary. On the privacy afforded by adaptive text compression. Computers and Security, 7:397$408,1988$.

[WNC87] Ian H. Witten, Radford Neal, and John G. Cleary. The c code that was printed in the COMM ACM 1987 article . ftp://ftp.cpsc.ucalgary.ca/pub/projects/ar.cod/, 1987. 